\title{
Identification of microtonal melodies: Effects of scale-step size, serial order, and training
}

\author{
RICHARD PARNCUTT and ANNABEL J. COHEN \\ Dalhousie University, Halifax, Nova Scotia, Canada
}

\begin{abstract}
The perception of microtonal scales was investigated in a melodic identification task. In each trial, eight pure tones, equally-spaced in log frequency in the vicinity of $700 \mathrm{~Hz}$, were presented in one of nine different serial orders. There were two experiments, each with 108 trials (six scales [tone sets] $\times$ nine serial orders $\times$ two repetitions). In each experiment, 30 subjects, half of whom were musically trained, were asked to match each melody to one of 9 visual representations (frequency-time grids). In Experiment 1, the six scales were spaced at intervals of 25, 33, 50, 67, 100, and 133 cents ( 100 cents $=1$ semitone $\sim 6 \%$ of frequency). Performance was worse for scale steps of 25 and 33 cents than it was for wider scale steps. There were no significant effects at other intervals, including the interval of 100 cents, implying that melodic pattern identification is unaffected by long-term experience of music in 12-tone equally tempered tuning (e.g., piano music). In Experiment 2, the six scales were spaced at smaller intervals, of $10,20,30,40,50$, and 60 cents. Performance for the three narrower scale steps was worse than that for the three wider scale steps. For some orders, performance for the narrowest scale step (10 cents) did not exceed chance. The smallest practical scale step for short microtonal melodies in a pattern-identification task was estimated as being 10-20 cents for chance performance, and 30-40 cents for asymptotic performance.
\end{abstract}

Most western music is based on diatonic scales, comprising 7 tones or pitch categories per octave, separated by intervals of one or two semitones. In modern usage, it is convenient to regard the 7-tone diatonic scales as subsets of the 12-tone chromatic scale, in which the pitch interval between adjacent tones is approximately constant (one semitone). When the 12 semitones are equal in size, corresponding to a frequency ratio of $2^{1 / 12}$, the chromatic scale is said to be equally tempered.

The equal spacing of the chromatic scale enables unlimited modulation and transposition of chromatic pitch patterns. The scale can also be musically limiting, in two ways. First, the scale limits the number of pitches available for composition or performance. Second, the equally tempered chromatic scale does not allow for variations in intonation, such as the difference between pure or just intonation (e.g., 5:4, or 3.86 semitones, for the major third) and Pythagorean intonation (81:64, or 4.08 semitones, for the same interval).

\footnotetext{
This research was supported by an operating grant from the Natural Sciences and Engineering Council of Canada (NSERC) to A.J.C. R.P received an International Fellowship from NSERC. The authors would like to thank Bradley Frankland for detailed comments on an earlier draft of the paper, Douglas Mewhort for the use of ANOVA7, J. O. Ramsay for the use of Multiscale II, and Adrian Houtsma and Robert Crowder for helpful suggestions during the review process. Parts of this study were presented to the Canadian Society for Brain, Behaviour, and Cognitive Science, Quebec City, 1992. Correspondence and requests for reprints may be addressed to R. Parncutt, Department of Psychology, Keele University, Keele, Staffordshire ST5 5BG (e-mail: r.parncutt@keele.ac.uk), or to A. J. Cohen, Department of Psychology, University of Prince Edward Island, 550 University Ave., Charlottetown, P.E.I., Canada C1A 4P3 (e-mail: annabel@ernie.psyc.upei.ca).
}

Microtonal intervals may be defined as pitch intervals that are smaller than one semitone, or that involve noninteger multiples of one semitone (e.g., 3.5 semitones); and microtonal scales are musical scales in which the distance between adjacent tones is consistently smaller than one semitone. Microtonal scales allow for greater numbers of pitch combinations than the chromatic scale. Moreover, equally tempered microtonal scales can approximate certain frequency ratios more closely than 12tone equal temperament (for examples, see Fuller, 1991). A microtonal melody may be defined for the purpose of this paper as a series of tones spanning microtonal intervals, or a series of tones from a microtonal scale.

The theory, practice, and notation of microtonal scales in Western music have been reviewed by Read (1990). The most commonly advocated (and used) microtonal subdivisions are quarter tones, which have been experimented with since the seventeenth century or earlier. However, despite this long history of experimentation, microtonal music remains uncommon.

The failure of microtonal scales to capture the attention of mainstream western musicians and audiences may be explicable in terms of categorical perception. Musicians are sometimes remarkably insensitive to microtonal deviations from 12-tone equal temperament in isolated intervals. Burns and Ward (1978) and Siegel and Siegel (1977a, 1977b) presented musicians with isolated tone pairs and asked them to estimate the size of the interval between them. Listeners tended to allocate all intervals to perceptual categories of width of approximately one semitone. For example, an interval of 5.3 semitones was usually perceived as a perfect fourth ( 5 semitones). 
Musicians are more sensitive to microtonal pitch variations in intervals presented in diatonic or chromatic contexts. Jordan (1987) replicated the key profiles of Krumhansl and Kessler (1982) by presenting a tonal context tuned to 12-tone equal temperament followed by a probe tone whose tuning was not necessarily equally tempered. He found that the profiles could be elaborated by the addition of quarter tones $(0.5$ semitone, or 50 cents) to the chromatic scale; however, eighth tones ( 25 cents) carried no new tonal information. Wapnick, Bourassa, and Sampson (1982) examined the perception of 10-tone diatonic melodies in which the first nine tones were tuned to $12-$ tone equal temperament and the tuning of the last tone was varied. They found that musicians were sensitive to intonational variations as small as 20 cents for tones in melodic contexts, and that they categorized to a lesser extent when intervals were presented in context rather than in isolation.

The present study investigates the perception of unaccompanied microtonal melodies by addressing the following four questions: (1) How "micro" can a microtonal scale be without destroying the identifiability of typical melodic fragments? In other words, what is the smallest practical interval size between adjacent scale steps? (2) What role does training play in the perception of microtonal melodies? How important is general musical training in comparison with training on the specific experimental task? (3) How important is the semitone interval in the perception of microtonal melodies? Are microtonal scales easier to perceive if they are based on integer fractions of a semitone (e.g., 50 cents, 33 cents), so that the interval of exactly one semitone occurs between certain pairs of pitches? And (4) how, and to what extent, does the perceptibility of microtonal melodies depend on melodic pattern? Melodic pattern may be defined as a combination of contour (the sequence of melodic ups and downs without regard to interval size, as defined by Dowling, 1978) and interval size relative to the prevailing scale-that is, the number of (microtonal) scale steps between successive tones, as opposed to actual interval sizes in semitones. The melodic patterns presented in the present study were created by varying the serial order of eight contiguous tones in equally spaced microtonal scales. In this study, the concept of melodic pattern is thus equivalent to the concept of serial order.

The next four sections survey literature pertinent to these questions.

\section{The Difference Limen for Frequency}

Regarding the smallest practical size of microtonal intervals (Question 1 above), the interval between scale steps must first of all exceed the just-noticeable difference (JND) or difference limen (DL) for the frequency of successive tones. The frequency DL for successive pure tones lies in the vicinity of $0.1 \%-0.2 \%$, or 2 3 cents, for tone durations exceeding about $100 \mathrm{msec}$ and frequencies in the range $100-5000 \mathrm{~Hz}$, increasing at lower and higher frequencies (Henning, 1966; Henning \& Forbes, 1967; Moore, 1973; Stücker, 1908; Wier, Je- steadt, \& Green, 1977) and at shorter tone durations (Fastl \& Hesse, 1984; Moore, 1973). The frequency DL for complex tones is somewhat smaller than that for pure tones; discrimination improves when harmonic overtones are added (Henning \& Grosberg, 1968; Moore, Glasberg, \& Shailer, 1984; Walliser, 1969).

Werner (1940) investigated the perception of a microtonal scale whose intervals spanned approximately $1 / 6$ semitone, or 17 cents. If Werner's subjects could indeed discriminate scale steps at such small intervals, the value of 17 cents may be taken as an initial estimate of the smallest practical microtonal interval. The smallest microtonal intervals in common use are 33-50 cents (Read, 1990).

Clearly, the smallest practical musical interval considerably exceeds the corresponding frequency DL. The following three possible reasons may be identified for the discrepancy: (1) Familiarity with musical scales. The above-mentioned studies on categorical perception confirm that the chromatic scale is familiar to western listeners. The farther one deviates from the equally tempered norm, the harder it may become to discriminate scale steps. (2) Training and subject selection in DL experiments. The frequency DL varies considerably as a function of listener and training, with differences typically spanning a factor of the order of ten among normally hearing subjects (Fastl \& Hesse, 1984). Both musical training and experimental training can enhance frequency acuity. Spiegel and Watson (1984) found that musicians (members of a professional symphony orchestra with no experience of psychoacoustical experiments) had frequency JNDs of between $0.1 \%$ and $0.45 \%$ (2-8 cents)-about three times smaller than those for nomusicians (first-year psychology majors without musical training). However, thresholds for nonmusicians with substantial training in frequency discrimination tasks were lower again (see also the data of Harris, 1952). A DL as small as $2-3$ cents is obtained only from the most sensitive and trained subjects; to be perceptible by a general musical audience, microtonal intervals must considerably exceed the minimum frequency DL obtained in psychoacoustical experiments. (3) Melodic context. Watson, Kelly, and Wroton (1976) determined JNDs for pairs of tone sequences that differed in the tuning of one of the tones. These JNDs were greater (worse) than those for isolated pairs of tones. The stimuli of Watson et al. varied in the amount of perceptual information they contained (stimulus uncertainty) and in the number of comparisons that listeners were required to make. In general, the greater the stimulus uncertainty or number of comparisons, the greater was the JND. The deterioration of performance in context may be understood as a result of a division of attention among the various tones, the intervals between them, and more global features such as contour.

\section{Training - Prior to and During the Experiment}

We anticipated that musicians would be considerably better than nonmusicians at identifying microtonal pitch 
patterns, since musical training can reduce the frequency DL (as indicated above). We also expected improvement in performance during the experiment itself. Werner (1940) found that the apparent size of microtonal intervals increased with increasing exposure to melodies composed from them, until they eventually "acquired the subjective character of a semi-tone" (p. 151). This anecdotal finding is consistent with the improvement of discrimination performance with training in a wide variety of perceptual tasks, as documented by Gibson (1953).

Strictly speaking, it is impossible to demonstrate conclusively a causal link between musical training and performance at musical or auditory tasks. Nevertheless, abundant evidence may be found for a link of some kind between musical training and musical performance (e.g., Brady, 1970; Cohen, 1994; Cohen, Lamoreux, \& Dunphy, 1989; Cuddy, 1970; Cuddy \& Cohen, 1976; Ericsson, Krampe, \& Tesch-Romer, 1993; Lymburner \& Cohen, 1994; Morrongiello, 1992; Parncutt, 1989, pp. 106, 115, 120, 131; Roberts, 1986; Sloboda, Davidson, \& Howe, 1994).

\section{The Equally Tempered Semitone}

Contemporary western listeners are repeatedly exposed to music performed on keyboard instruments tuned to 12-tone equal temperament. The familiarity of the equally tempered chromatic scale suggests that the ease with which a microtonal melody is processed may depend on the relative number of equally tempered semitone intervals it contains. Thus, melodies from the 12-tone equally tempered chromatic scale should be easier to process than melodies whose underlying scale steps are slightly greater or less than a semitone (e.g., $2 / 3$ or $4 / 3$ semitone); and melodies from a quarter tone $(1 / 2$ semitone) scale, in which intervals containing even numbers of scale steps belong to 12-tone equal temperament, should be easier to process than music based on a scale of intervals of $1 / 3$ or $2 / 3$ semitones, in which intervals must contain multiples of three scale steps to belong to 12 -tone equal temperament.

\section{Melodic Pattern and Serial Order}

Previous research (Divenyi \& Hirsch, 1974; Nickerson \& Freeman, 1974; Warren \& Byrnes, 1975) has shown that some serial orders of tones are more easily identified than others. Ease of identification of a stimulus would appear to depend on both ease of encoding and stability of mental representation. In an experimental context, ease of encoding additionally depends on the degree to which a stimulus is confused with other stimuli included in the same experimental design.

Stimulus parameters influencing identification include contour complexity, rule-governedness, and familiarity (Cohen \& Frankland, 1990). A pattern is easier to encode, and has a more stable mental representation, if its contour is less complex, if the pattern is perceived to be more rule-governed, or if the pattern is more familiar. Unaccompanied melodies played within the confines of the chromatic scale can often be recognized or identified according to their contour (Dowling, 1978). The exact sizes of intervals appear to play a subordinate role in melodic recognition. Similar results may be expected for microtonal melodies. Contour complexity may be defined most simply as the number of changes of direction in the pitch contour of a melody. According to this definition, a simple rising or falling scale has a contour complexity of zero, and a pattern that rises and then falls (or falls and then rises) has a contour complexity of one. Melodic patterns with zero contour complexity (rising or falling scales or arpeggios) are identified more reliably than melodic patterns with contours that change direction (Cuddy \& Cohen, 1976; Divenyi \& Hirsch, 1974; Nickerson \& Freeman, 1974). However, due to the additional effects of rule-governedness and familiarity, it is not generally true that a pattern with greater contour complexity is harder to identify than one with less complexity.

Patterns may be confused with other, similar patterns. The nature and extent of such confusions depends on the selection of patterns - the set of patterns provided to the subject as possible responses to each trial. The degree to which two patterns are confused depends, among other things, on the similarity of their contours (Cohen \& Frankland, 1990; Dowling, 1978).

The more rule-governed patterns are, or are perceived to be, the easier they are to encode (Garner, 1974). Other, assentially synonymous, terms for rule-governedness are apparent logic, regularity, structuredness, internal repetitiveness, and predictability. The opposite of rulegovernedness may be described as apparent randomness, structural complexity, or strangeness. A highly rulegoverned pattern might be one that rises and falls in a regular and predictable fashion (e.g., CEDFEGFA, where $C$ is the lowest tone), as compared with a less regular pattern with the same contour complexity (e.g., CAFGDFEA, again with $\mathrm{C}$ as the lowest tone). Some theoretical notions relevant to this issue were discussed by Boltz and Jones (1986), Cohen, Trehub, and Thorpe (1989), Deutsch and Feroe (1981), Restle (1979), and Simon and Sumner (1968). However, a comprehensive, quantitative model of apparent rule-governedness of melodic patterns, and hence of recognition accuracy as a function of melodic pattern or serial order, does not yet exist. The present study aims to provide further data upon which such a model could be based.

Note that Boltz and Jones (1986) defined contour complexity to include predictability of reversals. Here, we favor a simpler, more objective definition of contour complexity that includes only the number of reversals, and regard predictability of reversals as an aspect of rulegovernedness.

Regarding familiarity, it is common experience that familiar or "overlearned" melodic patterns are more easily identified than unfamiliar patterns. However, due to the complexity and intractability of musical exposure, it would be difficult to model this parameter in a reliable, quantitative way, even for just one subject. 


\section{EXPERIMENT 1}

\section{Method}

Subjects. Thirty first-year psychology students (mean age $=$ 21 years, standard deviation $[S D]= \pm 5$ ) participated in the experiment and received a credit point as compensation. One half of them, designated musicians, had played a musical instrument for at least 6 years (mean number of years of training on main instrument $=9 \pm 4[21 \pm 12$ when summed over different instruments]). The other half, designated nonmusicians, had played musical instruments for less than one year, and most of them had not played at all (mean $=0.2$ years). Each group comprised $10 \mathrm{fe}$ males and 5 males. A few of the subjects in both training groups had limited experience in auditory perception experiments-1 or $2 \mathrm{~h}$ maximum involving the 12-tone equally tempered scale; this experience, however, was not of a kind that would be expected to influence their frequency DLs.

Apparatus. Pure tones were produced by a NeXT computer ( 16 bit, $44.2 \mathrm{kHz}$ sample rate; see Cohen et al., 1991) and presented binaurally over headphones in a sound-attenuated room. Responses were made via the computer keyboard.

Stimuli. Each melody comprised 8 pure tones, equally spaced in $\log$ frequency. Intervals between adjacent scale steps were 25,33 , $50,67,100$, or 133 cents, corresponding to frequency ratios of $1: 2^{1 / 48}, 1: 2^{1 / 36}, 1: 2^{1 / 24}, 1: 2^{1 / 18}, 1: 2^{1 / 12}$, and $1: 2^{1 / 9}$, respectively. Frequencies lay in the vicinity of $700 \mathrm{~Hz}$, the center of the spectral pitch dominance region, according to Terhardt, Stoll, and Seewann (1982). Frequencies also lay within the region of smallest relative frequency discrimination thresholds (roughly $500-5000 \mathrm{~Hz}$, and centered on 1000-2000 Hz, according to data of Henning \& Grosberg, 1968). The frequencies selected for Scale 2 (33-cent spacing) were the same as those of the lower 8 of the 9 tones investigated by Cohen $(1988,1994)$ in absolute identification studies. The fifth tone in each ascending scale was always $769 \mathrm{~Hz}$, or 33 cents lower than G5 in the standard equally tempered scale (with A4 = $440 \mathrm{~Hz}$ ). The actual frequencies in each scale are given in Table 1. Tones had equal amplitude, and were presented at a free-field equivalent level of $55 \mathrm{~dB}$ (SPL). The duration of the tones was $200 \mathrm{msec}$, with $50 \mathrm{msec}$ rise and decay time. The silent intervals between tones lasted $200 \mathrm{msec}$, so that interonset times were $400 \mathrm{msec}$, and the total duration of each sequence was $3 \mathrm{sec}$.

Procedure. The subjects were tested individually in an Industrial Acoustics sound-attenuated room. The test session was preceded by a practice session. The practice began with the presentation of an ascending sequence of 8 tones at 67-cent intervals (Scale 4). Then the response panel (Figure 1) appeared (see Cohen et al., 1991), and the subject was told that the scale just heard corresponded to Pattern 1 on the panel. Order 1 was included in the experiment as a control against which results for other patterns could be compared; it also facilitated comparison with previous studies using the same set of orders, and encouraged subjects who found the task difficult.

Next, there were 9 practice trials, in which all 9 different melodic patterns (serial orders) on the response panel were presented. Only one of the six scales-that using 67-cent intervals-was used dur-

Table 1

Frequencies of Tones Presented in Experiment 1, in Hertz (Rounded)

\begin{tabular}{cccccccccc}
\hline Scale & Cents & & & & & & & \\
\hline 1 & 25 & 726 & 736 & 747 & 758 & 769 & 780 & 791 & 803 \\
2 & 33 & 712 & 726 & 740 & 754 & 769 & 784 & 799 & 815 \\
3 & 50 & 685 & 705 & 726 & 747 & 769 & 792 & 815 & 839 \\
4 & 67 & 659 & 685 & 712 & 740 & 769 & 799 & 831 & 863 \\
5 & 100 & 610 & 647 & 685 & 726 & 769 & 815 & 863 & 915 \\
6 & 133 & 565 & 610 & 659 & 712 & 769 & 831 & 897 & 969 \\
\hline
\end{tabular}

ing the practice session. The start of each melody was triggered by pressing a key. The melody was repeated five times, with silent intervals of $1,000 \mathrm{msec}$ between repetitions. The subjects attempted to recognize the pitch pattern of the melody by pressing a key corresponding to one of nine patterns displayed on the computer screen. The keys on the keyboard were arranged in a $3 \times 3$ grid that corresponded to the $3 \times 3$ grid of visual representations. It was possible to respond both during and after presentation of the melody. Visual feedback after each response indicated whether the response was correct. During the practice session, the experimenter also indicated which melodic pattern had actually been presented, by pointing to the screen.

Following the practice trials, the experimenter called up the test-trial panel and left the subject to perform the experiment alone. There followed two blocks of 54 trials. In each block, nine different serial orders (melodic patterns) of six different scales were presented, in a unique random order for each subject. After the experiment, the subjects completed a questionnaire on their musical background.

The experimental design had one between-subject variable (training level: musician vs. nonmusician) and three withinsubject variables (scale-step size [six], serial order [nine], and block [two]). The data, in this design, were submitted to an analysis of variance (ANOVA).

\section{Results and Discussion}

Performance, in terms of percent correct identification, improved with practice, from $66 \%$ correct in Block 1 to $72 \%$ correct in Block $2[F(1,28)=20, p<$ $.0005]$. The improvement was significant both for musicians [from $79 \%$ to $85 \% ; F(1,14)=21, p<.001$ ] and for nonmusicians [from $52 \%$ to $60 \% ; F(1,14)=7.3, p<$ $.05]$. The overall effect of musical training was large [ $56 \%$ correct responses for nonmusicians, $82 \%$ for musicians; $F(1,28)=20, p<.0005$ ]. Thus, general musical experience obtained before the experiment was more beneficial than experience obtained during the experiment.

Results as a function of scale-step size are shown in Figure 2(a). Identification rates averaged over all subjects ranged from $59 \%$ for Scale 1 ( 25 cents) to $74 \%$ for Scale $6(133$ cents; $c$. the chance rate of $1 / 9=11 \%)$. Results varied as a function of scale-step size both for musicians $[F(5,70)=8.7, p<.0001]$ and for nonmusicians $[F(5,70)=5.3, p<.001]$. Success rates for Scale $1(25$ cents) were lower than those for wider scales both for musicians $[F(1,14)=14, p<.005]$ and for nonmusicians $[F(1,14)=20, p<.001]$. Results for Scale 2 (33 cents) were lower than those for larger intervals for all subjects $[F(1,28)=15, p<.001]$ and for musicians separately $[F(1,14)=29, p<.0005]$, but the effect for nonmusicians was insignificant. Results for Scales 3-6 (i.e., for 50 cents and beyond) approached an asymptotic performance level of $85 \%-90 \%$ correct for the musicians and about $60 \%$ correct for the nonmusicians. No interaction was found between scale-step size and musical training. No effect was observed at the interval of one semitone (100 cents).

Results as a function of serial order and training are shown in Figure 2(b). In terms of mean success rates, order had a greater effect on the results than any other variable in the design. The lowest mean result for one order was 


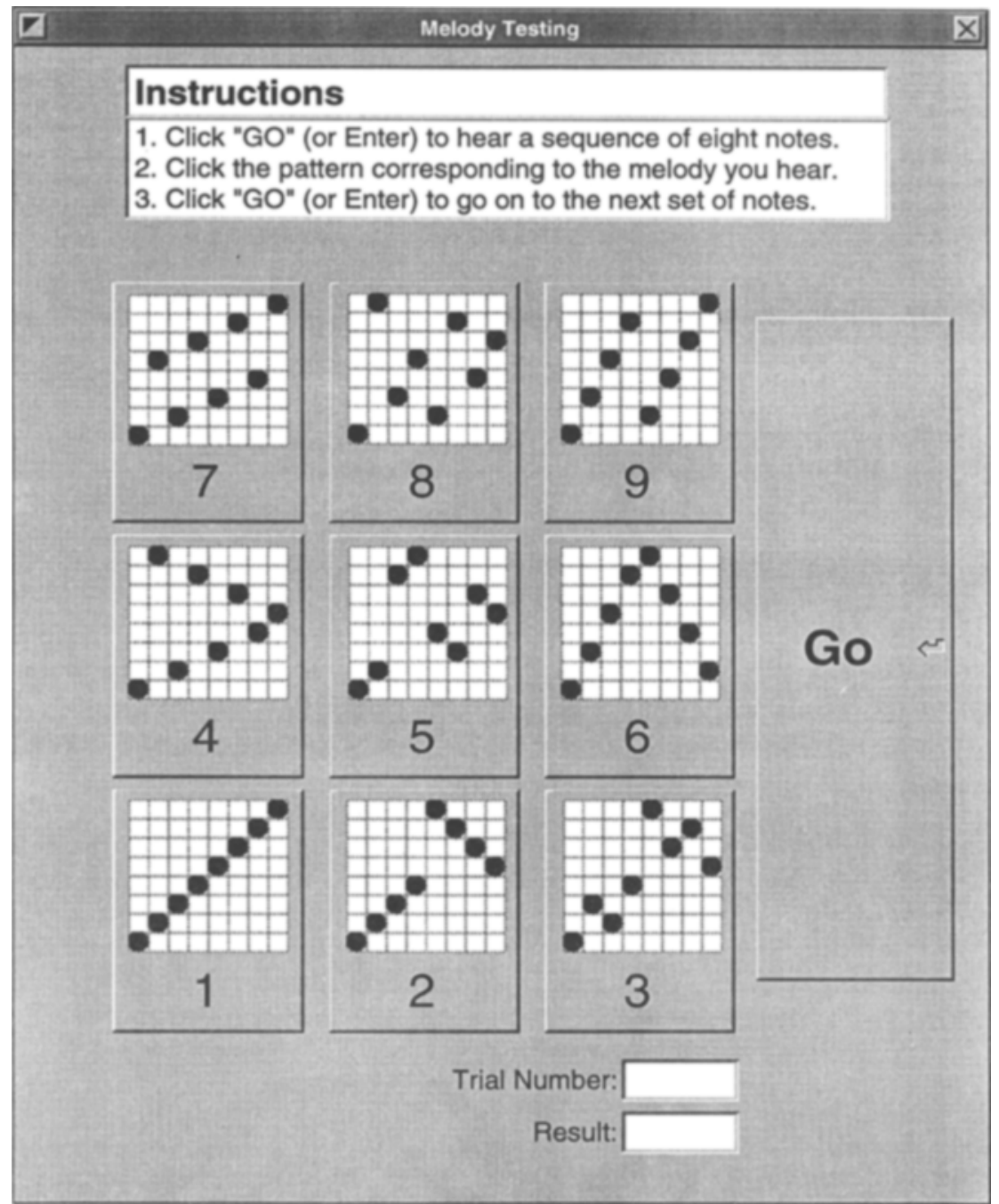

Figure 1. Response interface showing the nine serial orders (melodic patterns) used in the experiments.

$52 \%$, for Order 2 ; the highest was $93 \%$, for Order 1 . When Order 1 (the trivial case of a rising scale) is omitted, the results range from $52 \%$ for Order 2 to $74 \%$ for Order 7 .

The effect of order was highly significant both for musicians $[F(8,112)=10, p<.0001]$ and for nonmusicians $[F=13, p<.0001]$. Differences between results for specific orders were tested for significance by orthogonal contrasts within the ANOVA. Order 1, with the simplest contour (no changes of direction) was easiest to identify $[F(1,28)=82, p<.0001]$. Order 9 (two direction changes) was identified more reliably than Orders $3,4,5,7$, and 8 [all having more than two changes; $F(1,28)=5.9, p<.05]$. Order 5 (three direction changes) was easier to identify than Orders $3,4,7$, and 8 [all with more than three changes; $F(1,28)=11, p<.005]$. However, Orders 2 and 6 (one direction change) were no easier to identify than Orders $3,4,5,7,8$, and 9 (all of which had more than one change). Nor was Order 3 (five direction changes) easier to identify than Orders 4, 7, and 8 (all with more than five changes), presumably, in the case of Order 7, because this order was perceived as more predictable (rule-governed) than Order 3.

Orders with the same number of direction changes (e.g., Orders 4, 7, and 8, each with six direction changes) often differed regarding their ease of identification. Order 7 was easier to identify than Order $4[F(1,28)=16$, $p<.001]$, again suggesting that Order 7 sounded particularly rule-governed. Order 8 was harder to identify than Orders 4 and $7[F(1,28)=14, p<.005]$, presumably because of its apparent randomness (lack of rule-regularity). Order 6 (one direction change) was easier to identify than Order $2[F(1,28)=35, p<.0001]$, because (1) Order 2 was mistaken for Order 6 more often than Order 6 was mistaken for Order 2 ( 85 vs. 76 times); and (2) Order 2 was mistaken for Orders 3 and 4 (20 and 15 times, respectively) more often than Order 6 was (6 and 2 times).

Although Figure 2(a) indicated a gradual improvement in performance with increasing interval size, this 

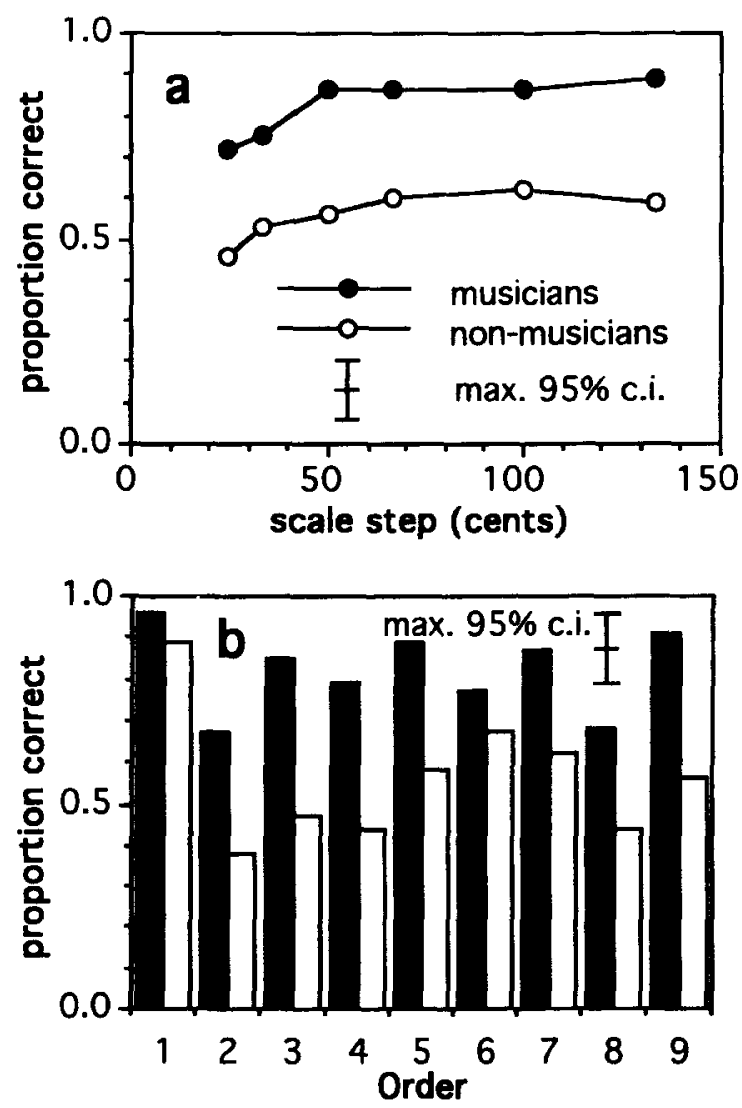

Figure 2. Overall proportion of correct responses in Experiment 1 for musicians (black circles and bars) and nonmusicians (white circles and bars), showing maximum $95 \%$ confidence interval. (a) $R e-$ sults as a function of scale-step size ( 270 responses per point). (b) Results as a function of order (180 responses per bar).

was not true for all orders, as shown in Figure 3. There was a significant interaction between scale-step size and serial order both for musicians $[F(40,560)=2.7, p<$ $.0001]$ and for nonmusicians $[F(40,560)=2.0, p<$ $.001]$. For example, identification rates for Order 6 exceeded rates for Order 2 , for Scale 1 in comparison with wider scales $[F(1,28)=12, p<.05]$, and identification rates for Order 9 exceeded rates for Orders 3, 4, 5, 7, and 8 , for Scale 2 in comparison with wider scales $[F(1,28)=$ $7.2, p<.05]$ and for Scale 3 in comparison with wider scales $[F(1,28)=5.5, p<.05]$.

Two tentative explanations may be advanced to explain these effects. First, adjacent tones in the narrower scales may often have been perceived as having the same pitch (or as belonging to the same pitch category), resulting in higher identification rates for orders containing few adjacent tones (such as Orders 6 and 9, in which successive tones are mostly two scale steps apart) in comparison with orders containing many adjacent tones (such as Orders 2 and 5). When Orders 6 and 9 were presented in the narrower scales, nonadjacent tones in the scale may have been interpreted as adjacent, effectively doubling the size of the scale step and increasing identi- fiability. The second explanation involves sensitivity to rule-governedness, or its opposite, randomness. The results presented in Figure 3 for Order 8, the most random, or least regular, order in the set, suggest that the quality of randomness was relatively hard to recognize for the narrower scales. In fact, results for Order 8 increased more steeply with wider scales than they did for Orders 4 and 7 , both for Scale 1 ( 25 cents) in comparison with wider scales $[F(1,28)=10, p<.005]$ and for Scale $2(33$ cents) in comparison with wider scales $[F(1,28)=24$, $p<.0005$ ].

Multidimensional scaling analysis. To further investigate relationships between orders, a multidimensional scaling (MDS) analysis (Ramsey, 1986) was performed on the $9 \times 9$ matrix of confusions between orders. Since in the ANOVA order had interacted with training $[F(8,224)=4.2, p<.0005]$, the MDS analysis was performed separately for musicians and nonmusicians. Results are shown in Figure 4 in the form of two-dimensional maps. In both parts of the figure, Order 2 is close to Order 6, and Order 4 to Order 8, reflecting frequent confusions between these pairs of orders. The confusion between Orders 2 and 6 was apparently due to their similar contours. Orders 4 and 8 probably sounded similarly random (nonrule-governed), due to their combination of high contour complexity and high mean successive interval size. In both figures, Order 1 is close to Order 9 (both are primarily composed of rising scale segments), Order 3 is close to Order 5 (both are characterized by many small intervals and frequent changes of direction), and Order 7 is close to Orders 4 and 8 (all have high contour complexity, or many changes of direction).

As a rule, orders near the top of the two parts of the figure have higher contour complexity (number of direction changes) than do those near the bottom. Contour complexity would thus represent an appropriate vertical axis label. However, Orders 3 and 5 conspicuously fail to conform to this scheme; Order 3 has higher contour complexity than Order 5, but is placed lower in both parts of the figure. An appropriate horizontal axis label might be "degree to which a pattern falls in pitch toward the end." Orders in the left halves of the figures (i.e., Orders 1, 7, 9, and 8) have an overall rising tendency; even Order 4 rises more than it falls, when intervals are added vectorially. Orders in the right halves of the figures (i.e., Orders $3,5,2$, and 6) tend to fall toward the end; this effect is greatest for orders on the far right (i.e., Orders 2 and 6).

\section{EXPERIMENT 2}

The results of Experiment 1 provided tentative answers to Questions 2 and 3 posed in the introductory section. Regarding Question 2, prior musical experience had a considerably greater beneficial effect on identification rates than did experience during the experiment. Regarding Question 3, no effects were found at the interval of one semitone, suggesting that there is nothing special about this interval in the perception of microtonal melodies. The results of Experiment 1 also pro- 


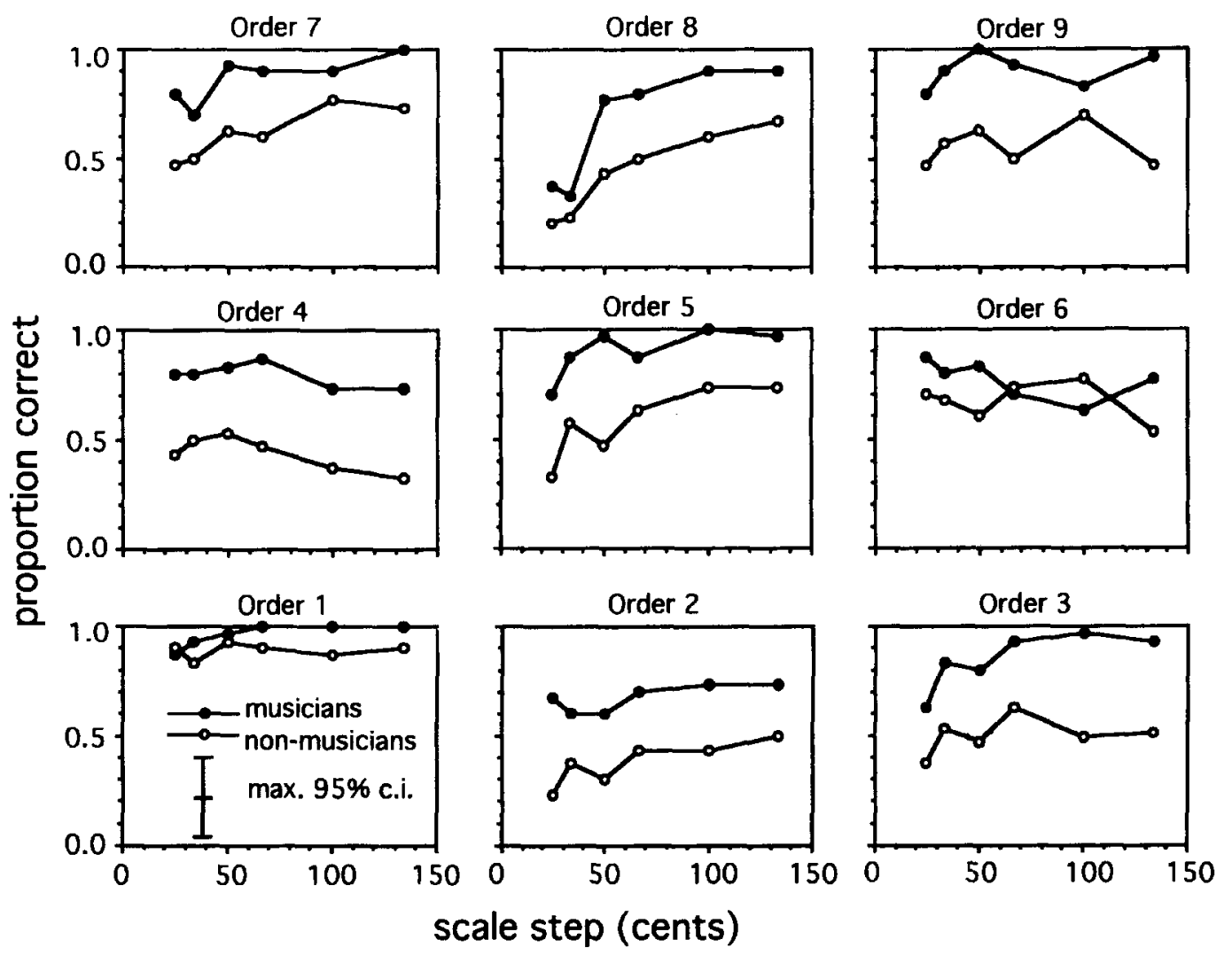

Figure 3. Proportion of correct responses in Experiment 1 for all combinations of scale step and order (30 responses per point). The arrangement of the panels corresponds to the arrangement of the orders on the response interface (Figure 1).

vided data that may contribute to an understanding of Question 4: The effect of melodic pattern appeared to depend on a number of separate factors, identified as contour complexity, rule-governedness, successive interval size relative to the prevailing scale, and context.

The results of Experiment 1 failed to answer Question 1, concerning the smallest practical interval in microtonal scales. As shown in Figure 2(a), the results approached asymptotic values for both musicians and nonmusicians at scale-step sizes greater than about 50 cents, implying that the sensory discriminability only affected results for scale steps of 50 cents or less. However, results for the narrowest scale of 25 cents (see Figure 3 ) were still consistently higher than the chance level of $11 \%$ ( 1 in 9) for all patterns.

In Experiment 2, we addressed this issue by investigating a narrower set of scales. Casual experimentation suggested that the interval of 10 cents was too small to allow melodic patterns to be recognized. We thus chose this as the smallest interval in the new set. It was also felt desirable to include one interval from the set used in Experiment 1 for comparison. The new set was 10, 20,30, 40,50 , and 60 cents, where the interval in common with Experiment 1 was 50 cents.

\section{Method}

Subjects. The subjects were drawn from the same subject pool as those in Experiment 1. No subject participated in both experiments. The number and mean level of musical experience of the subjects in each group (musicians and nonmusicians) were essentially the same as for those in Experiment 1. Moreover, the subjects' age range, gender ratio, and experience (or lack of experience) in psychoacoustical experiments were essentially the same as before.

Apparatus. The apparatus was the same as that used in Experiment 1 .

Stimuli. Each melody comprised eight pure tones, equally spaced in $\log$ frequency. Intervals between adjacent scale steps were $10,20,30,40,50$, and 60 cents, corresponding to frequency ratios of $1: 2^{1 / 120}, 1: 2^{1 / 60}, 1: 2^{1 / 40}, 1: 2^{1 / 30}, 1: 2^{1 / 24}$, and $1: 2^{1 / 20}$, respectively. The frequency of the fifth tone in each ascending scale was always $740 \mathrm{~Hz}$, corresponding to the musical tone FH5. The actual frequencies in each scale are given in Table 2. Levels and durations were the same as in Experiment 1.

Procedure. The procedure was the same as that used in Experiment 1 , with the exception that the practice session used a 50cent scale (Scale 5 in this experiment) rather than a 67 -cent scale (Scale 4 in Experiment 1).

\section{Results and Discussion}

As in Experiment 1, there was a large main effect of musical training [ $55 \%$ vs. $73 \% ; F(1,28)=14, p<.005]$. Performance again improved with increasing scale-step 

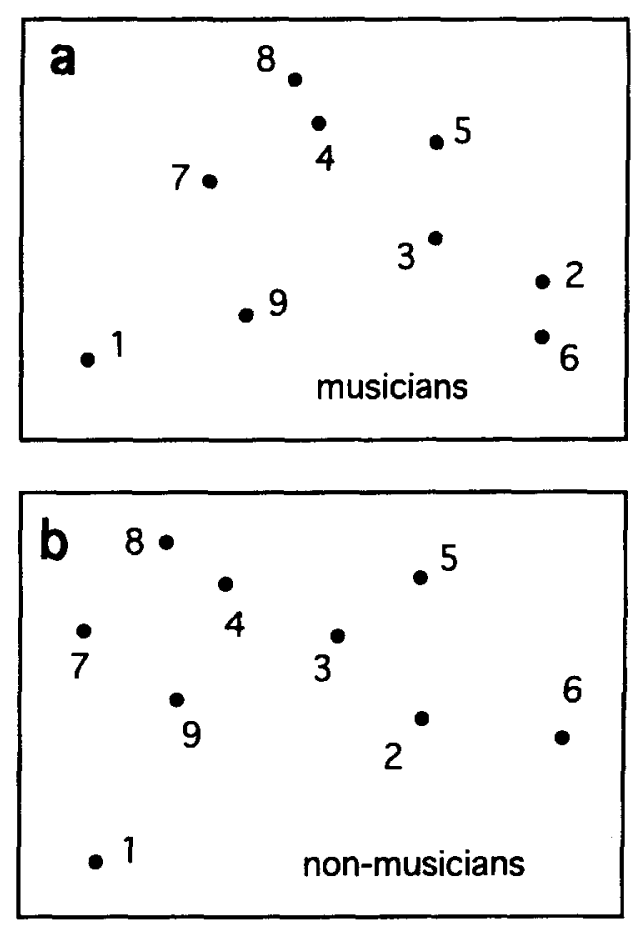

Figure 4. Multidimensional scaling solution for responses in Experiment 1 based on confusions between the nine serial orders shown in Figure 1 (180 responses per point). (a) Musicians. (b) Nonmusicians.

size both for musicians $[F(5,70)=40, p<.0001]$ and for nonmusicians $[F(5,70)=21, p<.0001]$, as shown in Figure 5(a). Mean results for each scale-step size ranged from $38 \%$ for Scale 1 (10 cents) to $74 \%$ for Scale 5 (50 cents). All results significantly exceeded the chance level of $1 / 9=11 \%$.

Performance on Scale 1 (10 cents) was worse than performance on other scales both for musicians $[F(1,14)=200, p<.0001]$ and for nonmusicians $[F(1,14)=41, p<.0005]$. Similarly, results for Scale 2 (20 cents) were worse than results for wider scales both for musicians $[F(1,14)=14, p<.005]$ and for nonmusicians $[F(1,14)=43, p<.0005]$. Results for Scale 3 (30 cents) were worse than results for wider scales for all listeners $[F(1,28)=6.3, p<.05]$ and for musicians $[F(1,14)=6, p<.05]$, but not for nonmusicians. Results for Scales 4-6 (40-60 cents) did not differ significantly from each other, implying that asymptotic performance (some $80 \%-85 \%$ for musicians, and roughly $65 \%$ for nonmusicians) was reached at an interval of 30-40 cents. As before, there was no interaction between scalestep size and musical training.

The main effect of order, shown in Figure 5(b), was again significant both for musicians $[F(8,112)=8.6$, $p<.0001]$ and for nonmusicians $[F(8,112)=6.4, p<$ $.0001]$. Overall performance improved from $61 \%$ in Block 1 to $67 \%$ in Block $2[F(1,28)=17, p<.001]$. A new interaction was observed between block and training $[F(1,28)=6.9, p<.05$; Figure $5 \mathrm{c}]$ : the improve- ment with practice between blocks was only significant for the nonmusicians [from $50 \%$ to $60 \% ; F(1,14)=17$, $p<.005]$.

The interaction between scale-step size and serial order (Figure 6) was significant for musicians $[F(40,560)=$ $1.8, p<.005$ ] but not for nonmusicians. In Order 3 (five direction changes), in comparison with Orders 4,7 , and 8 taken together (six direction changes), musicians' results for the two narrowest scales were worse than their results for wider scales [for Scale 1, $F(1,14)=4.8, p<$ .05 ; for Scale $2, F(1,14)=7.0, p<.05]$. This effect could be due to pitch discrimination: The successful identification of Order 3 requires the discrimination of smaller intervals between successive tones than does the identification of Orders 4, 7, and 8 . Note that the successful identification of Order 1 does not require discrimination of successive tones; its trivial contour may be recognized by comparing nonsuccessive tones. Musicians' results for Scale 1 (10 cents) were particularly good in Order 1 , regardless of scale-step size $[F(1,14)=$ $32, p<.0005]$.

Referring to results for Scale 1 (10 cents) in Figure 6, the following combinations of order and musical training did not yield results that differed significantly ( $t$ test, $p<.05)$ from chance performance $(1 / 9=11 \%)$ : Order 2 for nonmusicians (23\%); Order 3 for both nonmusicians $(10 \%)$ and musicians $(23 \%)$; Order 6 for nonmusicians $(17 \%)$; Order 8 for both nonmusicians $(13 \%)$ and musicians (17\%); and Order 9 for nonmusicians (20\%). However, results for Scale 2 (20 cents) were significantly better than chance for all combinations of order and training. It may be concluded that the minimum practical microtonal scale-step size lies between 10 and 20 cents, for the present set of melodic patterns.

Comparison of Experiments 1 and 2. Is the discriminability of scale steps affected by context (i.e., by the size of scale steps in recently heard melodic patterns)? Experiments 1 and 2 had been designed to examine this question by including a quarter-tone scale (50 cents) in each-Scale 3 in Experiment 1, and Scale 5 in Experiment 2. Overall mean results for the 50-cent scale did not differ significantly between experiments (86\% in Experiment 1 vs. $84 \%$ in Experiment 2 for musicians; $55 \%$ vs. $65 \%$ for nonmusicians). Results as a function of order for the 50 -cent scale in the two different experimental contexts are compared in Figure 7. They correlate significantly both for musicians $(r=.88$, $p<.005)$ and for nonmusicians $(r=.69, p<.05)$. It may

Table 2

Frequencies of Tones Presented in Experiment 2, in Hertz (Rounded)

\begin{tabular}{cccccccccc}
\hline Scale & Cents & & & & & & & & \\
\hline 1 & 10 & 723 & 727 & 732 & 736 & 740 & 744 & 749 & 753 \\
2 & 20 & 707 & 715 & 723 & 732 & 740 & 749 & 757 & 766 \\
3 & 30 & 690 & 703 & 715 & 727 & 740 & 753 & 766 & 780 \\
4 & 40 & 675 & 690 & 707 & 723 & 740 & 757 & 775 & 793 \\
5 & 50 & 659 & 679 & 699 & 719 & 740 & 762 & 784 & 807 \\
6 & 60 & 644 & 667 & 690 & 715 & 740 & 766 & 793 & 821 \\
\hline
\end{tabular}



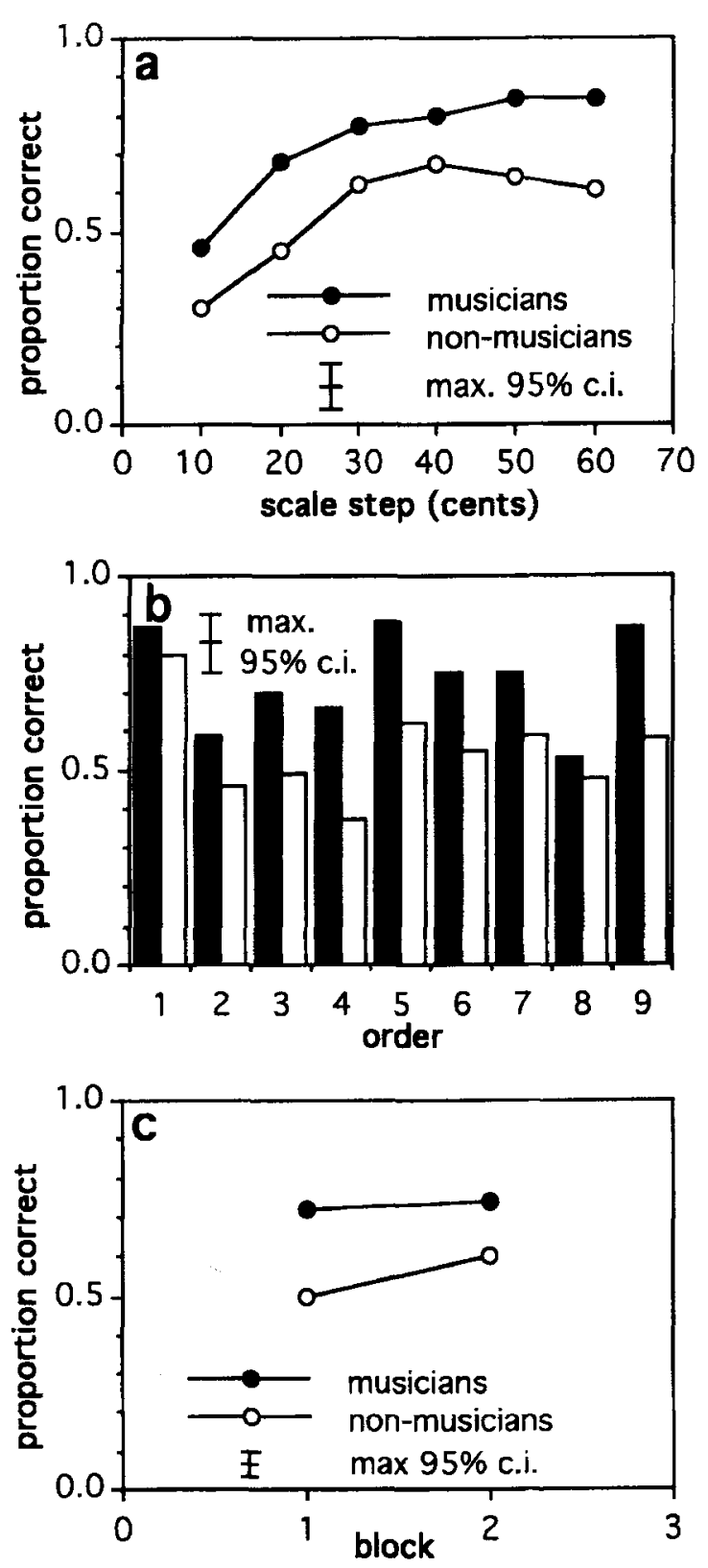

Figure 5. Overall proportion of correct responses in Experiment 2 for musicians (black circles and bars) and nonmusicians (white circles and bars), as a function of (a) scale-step size ( 270 responses per point), (b) order (180 responses per bar), and (c) block (810 responses per point).

be concluded that the difference between the two experimental contexts was too small to significantly affect results for the 50-cent scale.

Differences between the two experiments were further explored by entering all results into a single ANOVA. Because the scales in Experiment 2 were narrower, performance for the two narrowest scales in comparison with performance for the wider scales was worse than it was in Experiment 1 [Scale 1, $F(1,56)=32, p<.0001$; Scale $2, F(1,56)=4.9, p<.05]$, producing an interac- tion between scale-step size and experiment $[F(5,280)=$ $10, p<.0001]$. Moreover, effects of scale-step size and serial order were more independent in Experiment 2 (with narrower scales) than they were in Experiment 1, producing a three-way interaction between scale, order, and experiment $[F(40,2240)=1.6, p<.05]$.

\section{GENERAL DISCUSSION}

The absence of any effect at the semitone in Experiment 1 is reminiscent of the observed absence of any effect at the octave in similarity judgments of successive pairs of pure tones (Kallman, 1982; Parncutt, 1989) and at simple frequency ratios in consonance judgments of simultaneous pairs of pure tones (e.g., Plomp \& Levelt, 1965). Provided that subjects do not recognize musical intervals, the similarity of successive pure tones of equal SPL and duration simply declines as interval size increases. Pure tones appear to be no more similar when they span an octave than when they span a slightly larger or smaller interval (minor ninth, major seventh), despite the overwhelming importance of the octave interval in western music, and the familiarity of practically all westerners (including the subjects in the cited studies) with that music. Consistent with this idea, Houtsma (1968) found that the JND in interval size (frequency ratio) between two pure tones was independent of interval size, and was not enhanced if the interval approximated one octave; and Ward (1954) observed considerable intra- and interobserver variability in the size of the subjective octave.

Regarding the semitone interval, much western tonal music is performed on instruments tuned to, or close to, 12-tone equal temperament, a fact that may explain the preference for equal temperament over other temperaments (just, Pythagorean) often found in experiments on intonation (see Roberts, 1986, and references therein). In the present study, familiarity with 12-tone equal temperament failed to significantly influence experimental results. Our result is again consistent with the idea that in the absence of musical interval recognition, the similarity or relatedness of successive pure tones falls gradually and continuously as the pitch distance between them increases.

An effect at the semitone may possibly have been observed if complex tones instead of pure tones had been used in the experiments, for two reasons. First, given that almost all tones heard in tonal music are complex, and that the sound of equally tempered instruments such as pianos and other keyboards is familiar to most western listeners, the use of complex tones in the experiments may have helped listeners to draw on their musical experience whenever melodic patterns were confined to the equally tempered chromatic scale. Second, the pitches of the harmonic series (the octaves, fifths, major thirds, and so on, present in every harmonic complex tone) are quite closely matched by the equally tempered chromatic scale; this observation alone may explain why microtonal music is relatively uncommon. Pure tones 


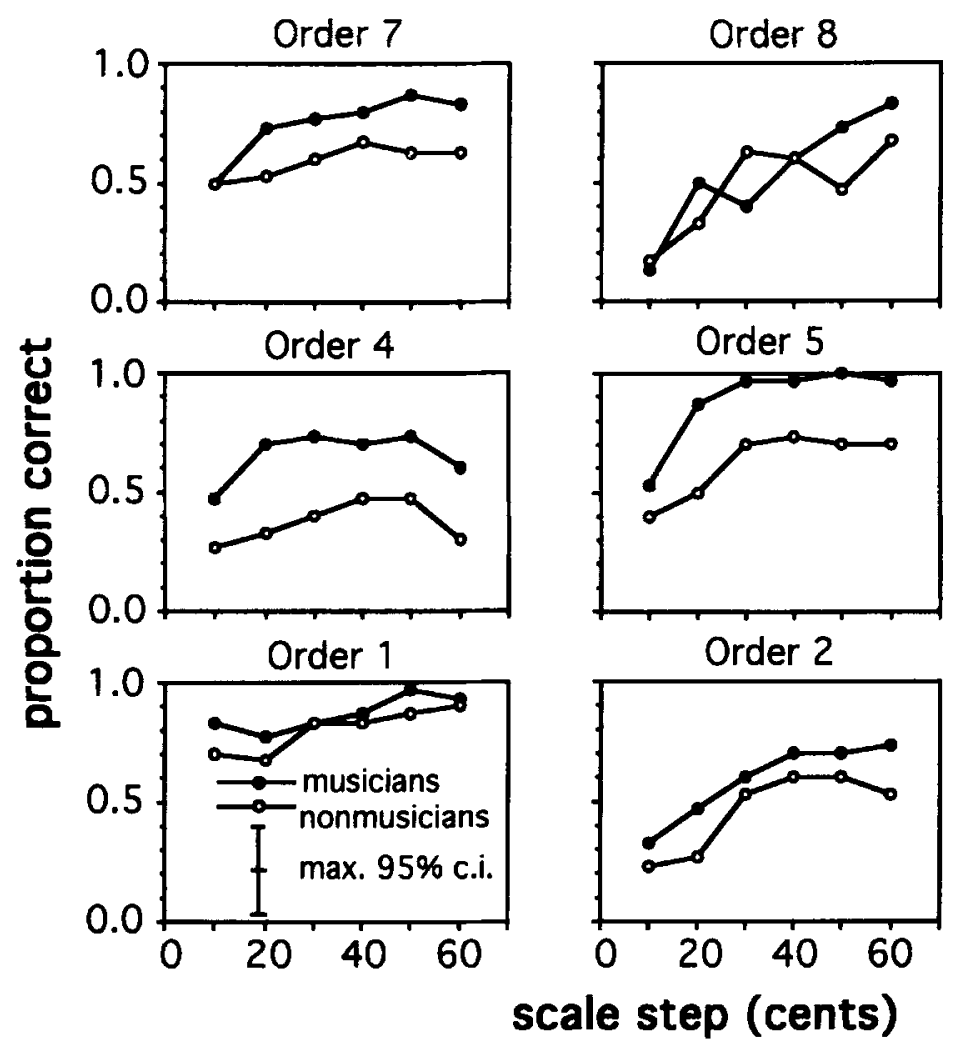

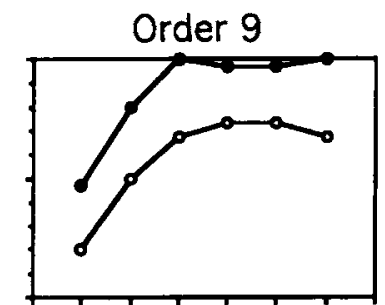

Order 6

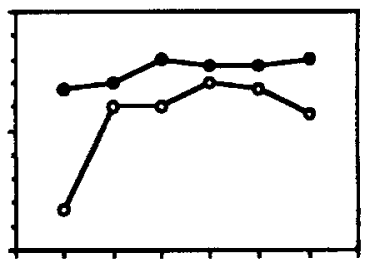

Order 3

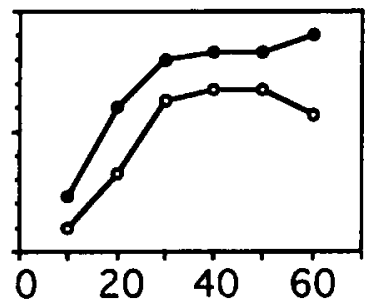

Figure 6. Proportion of correct responses in Experiment 2 for all combinations of scale step and onder (30 responses per point). The arrangement of the panels corresponds to the arrangement of the orders on the response interface (Figure 1 ).

were preferred in the present experiment because they are physically nonarbitrary, easy to reproduce in experimental settings, and perceptually unambiguous (whereas a complex tone may evoke more than one pitch). Future experiments may benefit from a comparison of results for pure and complex tones.

The results of the present experiments appear to have been influenced by the visual representations chosen for the orders included in the design. The graphs in Figure 1 are not necessarily ideal representations of the melodies, as Gestalt grouping principles (proximity, good continuation, and so forth) may operate rather differently in the visual and auditory modalities. For example, the up-and-down motion of Orders 4 and 7 is not immediately clear from the corresponding graphs. Identification rates for these orders could perhaps have been enhanced by joining temporally successive dots by straight lines, to emphasize the shape of the auditory contour. Confusion between Orders 2 and 6 may have been a result of the physical proximity of the dots in the visual representation of Order 2 (see Figure 1). Despite these drawbacks, the method of matching auditory to visual representations allows quicker and more spontaneous responses than, for example, notating pitches in an $8 \times 8$ grid, as in Cohen and Frankland (1990).

The visual aspect of the experimental task may have contributed to the superior performance of the musicians. Listeners who can read music may be better than other listeners at matching auditory patterns to visual patterns. There are two other possible reasons for the superior performance of musicians: First, musicians tend to have smaller frequency JNDs than nonmusicians (as discussed in the introductory section); and second, musicians may have superior cognitive abilities related to the perception of sound in general.

Jordan and Shepard (1987) provided evidence that the unequally spaced tones of the major scale may be perceived to be equally spaced. In other words, listeners may perceive successive pitch intervals as more equal than they really are. This suggests that listeners are less sensitive to unequal spacing in pitch height than they are to unequal spacing in visual height. The frequent confusions between Orders 2 and 6 in the present study are consistent with this idea. The leap between the fourth and fifth tones in Order 2 may simply have sounded smaller than it looked. This would explain why Order 2 was so often mistaken for Order 6, in which the up-down shape of the pitch contour was more visually prominent - even though the gap between the fourth and fifth tones was actually (and visually) much smaller in Order 6 than it was in Order 2.

\section{CONCLUSION}

We summarize the main results of our investigation as follows. The first four points correspond to the four ques- 

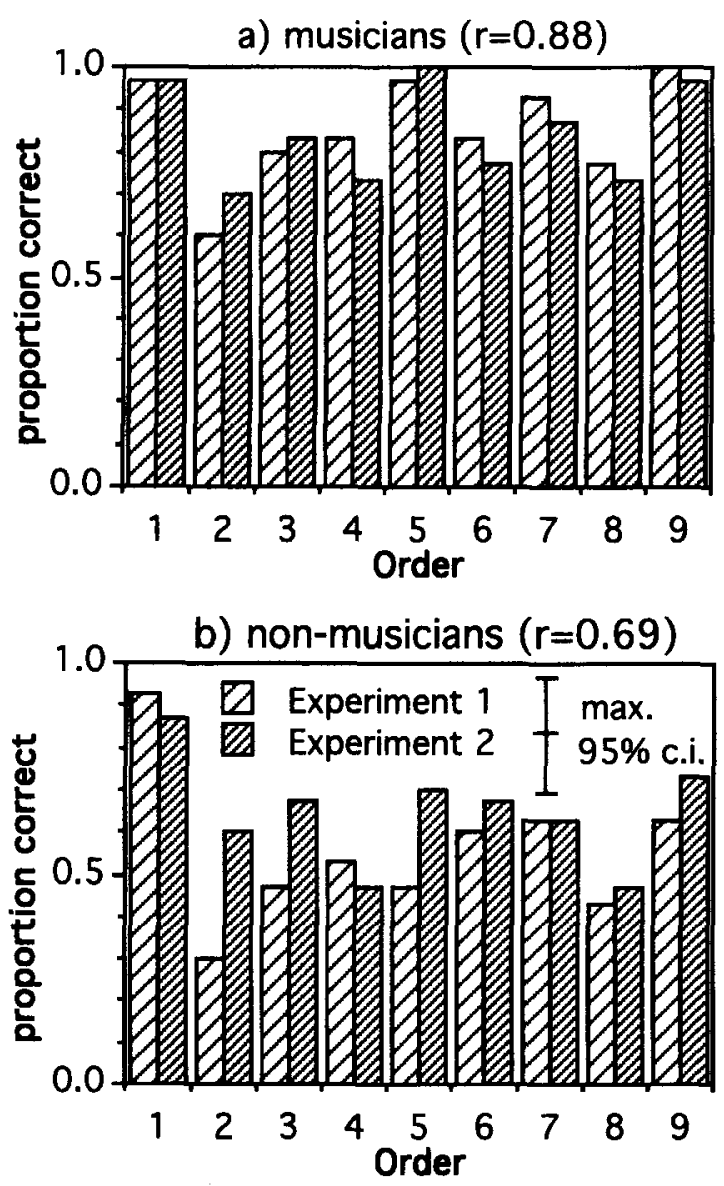

Figure 7. Comparison of results as a function of order for the quarter-tone scale (scale step $=\mathbf{5 0}$ cents) in two different contextsas Scale 3 in Experiment 1 (lighter bars), and as Scale 5 in Experiment 2 (darker bars) — (a) for musicians and (b) for nonmusicians (60 responses per bar).

tions posed in the introductory section. (1) The perceptibility of scale steps smaller than about 40 cents is limited by a combination of sensory and cognitive constraints. For the set of patterns used in our experiments, the threshold for the identification of short melodic patterns lies between 10 and 20 cents. (2) Both prior musical experience and learning during the described experiments improved success rates for identification of melodic patterns; however, the effect of musical experience was the greater of the two effects. (3) The equally tempered semitone played no role in our experiments, and so presumably does not influence the perception of unaccompanied microtonal melodies. (4) Serial-order effects in the identification of melodic patterns could be explained in a qualitative way in terms of contour complexity, rulegovernedness, successive ordinal interval size, and similarity of melodic patterns. (5) Independent effects of scale-step size and serial order accounted for a greater proportion of the variance of the success rates than did their interaction. The scale-by-order interaction was nevertheless significant in most cases. Two possible rea- sons for the interaction may be hypothesized: First, melodic patterns containing adjacent (close) scale steps in successive temporal positions may be particularly difficult to identify when realized in narrower scales, due to limitations of pitch discrimination; and second, patterns with intermediate degrees of rule-governedness may be more likely to be interpreted as random, or non-rule-governed, when realized in wider scales than they are when realized in narrower scales, due to stream segregation.

\section{REFERENCES}

Boltz, M., \& Jones, M. R. (1986). Does rule recursion make melodies easier to reproduce? If not, what does? Cognitive Psychology, 18, 389-431.

Brady, P. T. (1970). Fixed-scale mechanism of absolute pitch. Journal of the Acoustical Society of America, 48, 883-887.

BURNs, E. M., \& WARD, W. D. (1978). Categorical perceptionPhenomenon or epiphenomenon: Evidence from experiments in the perception of melodic musical intervals. Journal of the Acoustical Society of America, 63, 456-468.

CoHen, A. J. (1988, October). Context effects in the absolute judgment of a microtonal scale. Paper presented to the Canadian Psychological Association, Montreal; see also Canadian Psychology, 29, Abstract No. 145 .

CoHEN, A. J. (1994). Musical training and pitch schemata: Tonal influences in microtonal contexts. In I. Deliège (Ed.), Proceedings of the 3rd International Conference on Music Perception and Cognition, Liège, Belgium (pp. 81-82). Liège: European Society for the Cognitive Sciences of Music (ESCOM).

Cohen, A. J., \& FrankLand, B. F. (1990). Scale and serial order information in melodic perception: Independence or interdependence? Canadian Acoustics, 18, 3-10.

Cohen, A. J., LamoureuX, M. P., \& DunPhy, D. A. (1991). NeXT in the psychology laboratory: An example of an auditory pattern tracking task. Behavior Research Methods, Instruments, \& Computers, 23, 523-536

Cohen, A. J., Trehub, S. E., \& Thorpe, L. A. (1989). Effects of uncertainty on melodic information processing. Perception \& Psychophysics, 46, 18-28.

Cuddy, L. L. (1970). Training the absolute identification of pitch. Perception \& Psychophysics, 8, 265-269.

Cuddy, L. L., \& Cohen, A. J. (1976). Recognition of transposed melodic sequences. Quarterly Journal of Experimental Psychology, 28, 255-270.

DEUTSCH, D., \& FEROE, J. (1981). The internal representation of pitch sequences in tonal music. Psychological Review, 88, 503-522.

DivenYi, P. L., \& HiRsCH, I. J. (1974). Identification of temporal order in three-tone sequences. Journal of the Acoustical Society of America, 56, 144-151.

Dowling, W. J. (1978). Scale and contour: Two components of a theory of memory for melodies. Psychological Review, 85, 341-354.

Ericsson, K. A., Krampe, R. T., \& Tesch-Romer, C. (1993). The role of deliberate practice in the acquisition of expert performance. Psychological Review, 100, 363-406.

Fastl, H., \& Hesse, A. (1984). Frequency discrimination for pure tones at short durations. Acustica, 56, 41-47.

FUller, R. (1991). A study of microtonal equal temperaments. Journal of Music Theory, 35, 211-237.

GARNER, W. (1974). The processing of information and structure. New York: Wiley.

GIBSON, E. J. (1953). Improvement in perceptual judgments as a function of controlled practice or training. Psychological Bulletin, 50, $401-431$

HARRIS, J. D. (1952). Pitch discrimination. Journal of the Acoustical Society of America, 24, 750-755.

HenNing, G. B. (1966). Frequency discrimination of random amplitude tones. Journal of the Acoustical Society of America, 39, 336-339. 
HenNing, G. B., \& Forbes, S. M. (1967). Frequency discrimination as a function of frequency (Abstract). Journal of the Acoustical Society of America, 42, 1216.

HENNING, G. B., \& GRosBerg, S. L. (1968). Effect of harmonic components on frequency discrimination. Journal of the Acoustical Society of America, 44, 1386-1389.

Houtsma, A. J. M. (1968). Discrimination of frequency ratios (Abstract). Journal of the Acoustical Society of America, 44, 383.

JORDAN, D. S. (1987). Influence of the diatonic tonal hierarchy at microtonal intervals. Perception \& Psychophysics, 41, 482-488.

JoRDAN, D. S., \& ShePARD, R. N. (1987). Tonal schemas: Evidence obtained by probing distorted musical scales. Perception \& Psychophysics, 41, 489-504.

KALLMAN, H. J. (1982). Octave equivalence as measured by similarity ratings. Perception \& Psychophysics, 32, 37-49.

Krumhansl, C. L., \& Kessler, E. J. (1982). Tracing the dynamic changes in perceived tonal organization in a spatial representation of musical keys. Psychological Review, 89, 334-368.

Lymburner, J. A., \& Cohen, A. J. (1994). Accounting for musicians' superior auditory serial-order identification: Audition or notation? Canadian Acoustics, 22, 153-154.

MOORE, B. C. J. (1973). Frequency difference limens for short duration tones. Journal of the Acoustical Society of America, 54, 610-619.

MoOre, B. C .J., Glasberg, B. R., \& Shailer, M. J. (1984). Frequency and intensity difference limens for harmonics within complex tones. Journal of the Acoustical Society of America, 75, 550-561.

Morronglello, B. A. (1992). Effects of training on children's perception of music: A review. Psychology of Music, 20, 29-41.

Nickerson, R. S., \& FreEman, B. (1974). Discrimination of the order of the components of repeating tone sequences: Effects of frequency separation and extensive practice. Perception \& Psychophysics, 16, 471-477.

PARnCUTt, R. (1989). Harmony: A psychoacoustical approach. Berlin: Springer-Verlag.

Plomp, R., \& Levelt, W. J. M. (1965). Tonal consonance and critical bandwidth. Journal of the Acoustical Society of America, 38, 548560.

RaMSEY, J. O. (1986). Multiscale II manual. Montreal: McGill University, Department of Psychology.

READ, G. (1990). 20th-century microtonal notation. New York: Greenwood Press.

RESTLE, F. (1979). Serial pattern learning. Journal of Experimental Psychology, 83, 120-125.

RoBERTS, L. A. (1986). Consonance judgments of musical chords by musicians and untrained listeners. Acustica, 62, 163-171.
Siegel, J. A., \& Siegel, W. (1977a). Absolute identification of notes and intervals by musicians. Perception \& Psychophysics, 21, 143152.

Siegel, J. A., \& Siegel, W. (1977b). Categorical perception of tonal intervals: Musicians can't tell sharp from flat. Perception \& Psychophysics, 21, 399-407.

Simon, H. A., \& Sumner, R. K. (1968). Pattern in music. In B. Kleinmuntz (Ed.), Formal representations of human judgment (pp. 219250). New York: Wiley.

Sloboda, J. A., Davidson, J. W., \& Howe, M. J. A. (1994, August). Is everyone musical? The Psychologist, 7, 349-365.

SPIEGEL, M. F., \& Watson, C. S. (1984). Performance on frequencydiscrimination tasks by musicians and nonmusicians. Journal of the Acoustical Society of America, 76, 1690-1695.

STÜCKER, N. (1908). Über die Unterschiedsempfindlichkeit für Tonhöhen in verschiedenen Tonregionen [On differential pitch sensitivity in different registers]. Zeitschrift für Sinnesphysiologie, 42, 392-408.

Terhardt, E., Stoll, G., \& Seewann, M. (1982). Algorithm for extraction of pitch and pitch salience from complex tonal signals Journal of the Acoustical Society of America, 71, 679-688.

WALLISER, K. (1969). Zur Unterschiedsschwelle der Periodentonhöhe [On the difference threshold of periodicity pitch]. Acustica, 21, 329336.

WAPNICK, J., Bourassa, G., \& SAMPSON, J. (1982). The perception of tonal intervals in isolation and in melodic context. Psychomusicology, 2, 21-37.

WARD, W. D. (1954). Subjective musical pitch. Journal of the Acoustical Society of America, 26, 369-380.

WARREN, R. M., \& BYRNES, D. L. (1975). Temporal discrimination of recycled tonal sequences: Pattern matching and naming of order by untrained listeners. Perception \& Psychophysics, 18, 273-280.

WATSON, C. S., Kelly, W. J, \& WROTON, M. W. (1976). Factors in the discrimination of tonal patterns: II. Selective attention and learning under various levels of stimulus uncertainty. Journal of the Acoustical Society of America, 60, 1176-1186.

Werner, H. (1940). Musical "micro-scales" and "micro-melodies." Journal of Psychology, 10, 149-156.

Wier, C. C., Jesteadr, W., \& Green, D. M. (1977). Frequency discrimination as a function of frequency and sensation level. Journal of the Acoustical Society of America, 61, 178-184.

(Manuscript received January 26, 1994; revision accepted for publication January 30,1995 .) 